\title{
Implicit methods for the first derivative of the solution to heat equation
}

\author{
Suzan C. Buranay ${ }^{1 *}$ (D) and Lawrence A. Farinola
}

\author{
"Correspondence: \\ suzan.buranay@emu.edu.tr \\ ${ }^{1}$ Department of Mathematics, \\ Faculty of Arts and Sciences, Eastern \\ Mediterranean University, \\ Famagusta, North Cyprus, Turkey
}

\begin{abstract}
We propose special difference problems of the four point scheme and the six point symmetric implicit scheme (Crank and Nicolson) for the first partial derivative of the solution $u(x, t)$ of the first type boundary value problem for a one dimensional heat equation with respect to the spatial variable $x$. A four point implicit difference problem is proposed under the assumption that the initial function belongs to the Hölder space $C^{5+\alpha}, 0<\alpha<1$, the nonhomogeneous term given in the heat equation is from the Hölder space $C_{x, t}^{3+\alpha, \frac{3+\alpha}{2}}$, the boundary functions are from $C^{\frac{5+\alpha}{2}}$, and between the initial and boundary conditions the conjugation conditions up to second order $(q=0,1,2)$ are satisfied. When the initial function belongs to $C^{7+\alpha}$, the nonhomogeneous term is from $C_{x, t}^{5+\alpha, \frac{5+\alpha}{2}}$, the boundary functions are from $C^{\frac{7+\alpha}{2}}$ and the conjugation conditions up to third order $(q=0,1,2,3)$ are satisfied, a six point implicit difference problem is given. It is proven that the solution of the given four point and six point difference problems converge to the exact value of $\frac{\partial u}{\partial x}$ on the grids of order $O\left(h^{2}+\tau\right)$ and $O\left(h^{2}+\tau^{2}\right)$, respectively, where $h$ is the step size in spatial variable $x$ and $\tau$ is the step size in time. Theoretical results are justified by numerical examples.
\end{abstract}

MSC: $65 \mathrm{M} 06 ; 65 \mathrm{M} 12 ; 65 \mathrm{M} 22$

Keywords: Finite difference method; Approximation of derivatives; Uniform error; Heat equation

\section{Introduction}

In science, especially in mathematical physics, not only the calculation of the solution of the differential equation but also the calculation of the first and second derivatives of the solution is very important to provide information about some physical phenomena [1]. For example, Tuttle [2] in a theory of the drying wood adopts the fundamental hypothesis that the rate of which transfusion takes place transversely with respect to the wood fibers $\left(\frac{\partial \theta}{\partial t}\right)$ is proportional to the slope of the moisture gradient $\left(\frac{\partial^{2} \theta}{\partial x^{2}}\right)$, where $\theta$ is the moisture content expressed as a percentage of the oven-dry weight of the wood. Therefore, accurate approximation of $\frac{\partial \theta}{\partial x}$ is very important to provide information about the moisture gradient. Since the differentiation operation is ill-conditioned, to find a highly accurate approximation for the derivatives of the solution of a differential equation is problematic.

In [3] the uniform convergence of the difference derivatives over the whole grid domain to the corresponding derivatives of the exact solution for the two dimensional

(c) The Author(s) 2018. This article is distributed under the terms of the Creative Commons Attribution 4.0 International License (http://creativecommons.org/licenses/by/4.0/), which permits unrestricted use, distribution, and reproduction in any medium, provided you give appropriate credit to the original author(s) and the source, provide a link to the Creative Commons license, and indicate if changes were made. 
Laplace equation with order $O\left(h^{2}\right)$ is proved. In [4], under the conditions that the boundary functions belong to $C^{6, \lambda}, 0<\lambda<1$, on the sides of the rectangle and are continuous on the vertices and second, fourth order derivatives satisfy the compatibility conditions on the vertices which result from the Laplace equation, difference schemes are constructed for the first and pure second order derivatives of the solution. It is proved that the order of convergence of the solutions of these difference schemes is $O\left(h^{4}\right)$.

For the 3D Laplace equation on a rectangular parallelepiped, recent studies are given in [5] and [6] and the constructed difference schemes converge with the order of $O\left(h^{2}\right)$ and $O\left(h^{4}\right)$, respectively, to the first and pure second derivatives of the exact solution of the 3D Dirichlet problem. It is assumed in [5] that the fourth derivatives of the boundary functions on the faces of a parallelepiped satisfy the Hölder condition, and on the edges their second derivatives satisfy the compatibility condition, whereas in [6] they are assumed to have sixth order derivatives satisfying the Hölder condition on the faces, and their second and fourth order derivatives satisfy the compatibility conditions on the edges. Most recently, in [7] difference schemes on a cubic grid for obtaining the solution of the Dirichlet problem for the 3D Laplace equation on a rectangular parallelepiped, its first and pure second derivatives difference schemes are constructed and the approximate values of the first and pure second derivatives converge with orders $O\left(h^{6}|\ln h|\right)$ and $O\left(h^{5+\lambda}\right), 0<\lambda<1$, respectively. It is assumed that the boundary functions on the faces have seventh derivatives satisfying the Hölder condition and on the edges their second, fourth and sixth derivatives satisfy the compatibility condition. At the same time in [8], an $O\left(h^{p-1}\right), p \in[4,5]$ order of approximation for the first order derivatives of the solution of the 3D Laplace equation is proven under a weaker assumption on the smoothness of the boundary functions on the faces of the parallelepiped than those used in [6].

In this study special difference problems of four point and six point symmetric implicit difference schemes for the derivative of the solution $u(x, t)$ of the first type boundary value problem for one dimensional heat equation with respect to the spatial variable $x$ are proposed. For the construction of the four point implicit difference problem we require that:

(a) the initial function belongs to $C^{5+\alpha}$, the nonhomogeneous term given in the heat equation is from $C_{x, t}^{3+\alpha, \frac{3+\alpha}{2}}$, the boundary functions are from $C^{\frac{5+\alpha}{2}}$, and the conjugation conditions of orders $q=0,1,2$ are satisfied at the corners of the boundary. For the construction of the six point implicit difference problem it is assumed that:

(b) the initial function belongs to $C^{7+\alpha}$, the nonhomogeneous term is from $C_{x, t}^{5+\alpha, \frac{5+\alpha}{2}}$, the boundary functions are from $C^{\frac{7+\alpha}{2}}$, and the conjugation conditions of orders $q=0,1,2,3$ are satisfied.

The work is organized as follows: In Sect. 2, for the approximate solution of first type boundary value problem for one dimensional heat equation we use four point implicit or six point symmetric implicit schemes [9] under the assumption that the boundary value problem satisfies the conditions (a) or (b) respectively. In both cases for the error function we provide a pointwise prior estimation depending on $\Upsilon(x, t)$, which is the distance from the current grid point in the domain to the boundary. In Sect. 3, we consider the boundary value problem satisfying the conditions (a) and propose a special four point implicit difference problem for the approximation of $\frac{\partial u}{\partial x}$. We prove that the solution of the 
constructed difference scheme converges uniformly to the exact value of $\frac{\partial u}{\partial x}$ on the grids of order $O\left(h^{2}+\tau\right)$ where $h$ is the step size in spatial variable $x$ and $\tau$ is the step size in time. In Sect. 4 , we require that the boundary value problem satisfies the conditions (b) hence, a special six point implicit difference problem for the approximation of $\frac{\partial u}{\partial x}$ is proposed. Uniform convergence of order $O\left(h^{2}+\tau^{2}\right)$ for this scheme is shown. In Sect. 5, to justify the theoretical results numerical examples are constructed and obtained results are presented via tables and figures. In Sect. 6, concluding remarks are given.

\section{Implicit difference solution of first type boundary value problem for one dimensional heat equation}

\subsection{Basic notations and first type boundary value problem}

Based on Section 5, Chapter IV in [10], we give the following definitions. We denote by $\mathcal{L}\left(x, t, \frac{\partial}{\partial x}, \frac{\partial}{\partial t}\right)$ the linear parabolic differential operator with real coefficients

$$
\mathcal{L}\left(x, t, \frac{\partial}{\partial x}, \frac{\partial}{\partial t}\right) u \equiv \frac{\partial u}{\partial t}-\sum_{i, j=1}^{n} w_{i, j}(x, t) \frac{\partial^{2} u}{\partial x_{i} \partial x_{j}}+\sum_{i=1}^{n} w_{i}(x, t) \frac{\partial u}{\partial x_{i}}+w(x, t) u .
$$

Let $\Omega$ be a bounded domain in $n$-dimensional Euclidean space $E_{n}$. It is assumed that the coefficients of the operator of (1) are defined in a layer $D=E_{n} \times(0, T)$. In the cylindrical domain $Q=\Omega \times(0, T)$ with lateral surface $S_{T}$ or more precisely the set of points $(x, t)$ of $E_{n+1}$ with $x=\left(x_{1}, x_{2}, \ldots, x_{n}\right) \in S, t \in[0, T]$ where $S$ is the sufficiently smooth boundary of $\Omega$ and that $\bar{\Omega}=\Omega \cup S$, the first type boundary value problem is given as

$$
\begin{aligned}
& \mathcal{L}\left(x, t, \frac{\partial}{\partial x}, \frac{\partial}{\partial t}\right) u=f(x, t), \\
& \left.u\right|_{t=0}=\varphi(x), \\
& \left.u\right|_{S_{T}}=\phi(x, t) .
\end{aligned}
$$

Let $q$ be a non-negative integer. We use the notations

$$
\begin{aligned}
& u^{(0)}(x)=\varphi(x), \quad q=0, \\
& u^{(q)}(x)=\left.\frac{\partial^{q} u(x, t)}{\partial t^{q}}\right|_{t=0}, \quad q=1,2,3, \ldots
\end{aligned}
$$

and the operator

$$
\hat{A}\left(x, t, \frac{\partial}{\partial x}\right) u \equiv \sum_{i, j=1}^{n} w_{i, j}(x, t) \frac{\partial^{2} u}{\partial x_{i} \partial x_{j}}-\sum_{i=1}^{n} w_{i}(x, t) \frac{\partial u}{\partial x_{i}}-w(x, t) u .
$$

From (2), (5) and (7), Eq. (6) can be rewritten as

$$
\begin{aligned}
& u^{(1)}(x)=\hat{A}\left(x, 0, \frac{\partial}{\partial x}\right) \varphi(x)+f(x, 0) \\
& u^{(q+1)}(x)=\left.\left(\frac{\partial^{q}}{\partial t^{q}} \hat{A}\left(x, t, \frac{\partial}{\partial x}\right) u(x, t)+\frac{\partial^{q}}{\partial t^{q}} f(x, t)\right)\right|_{t=0}, \quad q=1,2,3, \ldots
\end{aligned}
$$


The conjugation (compatibility) conditions up to order $m \geq 0$ are

$$
\left.u^{(q)}(x)\right|_{x \in S}=\left.\frac{\partial^{q} \phi(x, t)}{\partial t^{q}}\right|_{t=0}=\phi^{(q)}(x), \quad q=0,1, \ldots, m .
$$

Let $\bar{Q}$ and $\bar{S}_{T}$ be the closure of $Q$ and $S_{T}$, respectively, and $s>0$ be a non-integer number. Further, $C_{x, t}^{s, / 2}(\bar{Q})$ denotes the classical Hölder space of functions $u(x, t)$ that are continuous in $\bar{Q}$ together with all derivatives of the form $\partial_{t}^{j 0} \partial_{x}^{j}$ for $2 j_{0}+j<s$ and have finite norm defined in $C_{x, t}^{s, s / 2}(\bar{Q}) . C^{s}(\bar{\Omega})$ is the Hölder space whose elements are continuous functions $g(x)$ in $\bar{\Omega}$ having in $\bar{\Omega}$ continuous derivatives up to order $[s]$ inclusively, and have finite norm defined in $C^{s}(\bar{\Omega})$ (see [10]).

Theorem 1 (From Theorem 5.2, Section 5, Chapter IV in [10]) Suppose $s>0$ is a noninteger number, the coefficients of the operator $\mathcal{L}$ belongs to the class $C_{x, t}^{s, \frac{s}{2}}(\bar{Q})$, and the boundary $S$ belongs to the class $C^{s+2}$. Then, for any $f \in C_{x, t}^{s, \frac{s}{2}}(\bar{Q}), \varphi(x) \in C^{s+2}(\bar{\Omega})$, and $\phi(x, t) \in C_{x, t}^{s+2, \frac{s}{2}+1}\left(\bar{S}_{T}\right)$ satisfying the compatibility conditions (10) up to order $\left[\frac{s}{2}\right]+1$, problem (2)-(4) has a unique solution from the class $C_{x, t}^{s+2, \frac{s}{2}+1}(\bar{Q})$.

\subsection{Implicit difference solution of one dimensional problem}

Take $\Omega=(0, b), \sigma_{T}=(0, T)$, and $\bar{\Omega}, \bar{\sigma}_{T}$ are the closure of these sets respectively, also $Q_{T}=$ $\{(x, t): 0<x<b, 0<t \leq T\}, \gamma_{1}=\left\{(0, t): t \in \bar{\sigma}_{T}\right\}, \gamma_{2}=\{(x, 0): x \in \bar{\Omega}\}$, and $\gamma_{3}=\{(b, t): t \in$ $\left.\bar{\sigma}_{T}\right\}$. Let $\gamma=\bigcup_{i=1}^{3} \gamma_{i}$ represent the boundary of $Q_{T}$, and $\bar{Q}_{T}=Q_{T} \cup \gamma$. We use the notations $\partial_{t}^{k}=\frac{\partial^{k}}{\partial t^{k}}, \partial_{x}^{k}=\frac{\partial^{k}}{\partial x^{k}}$ and $D_{t}^{k}=\frac{d^{k}}{d t^{k}}, D_{x}^{k}=\frac{d^{k}}{d x^{k}}$ to present the $k$ th partial and ordinary derivatives respectively with respect to time variable $t$, spatial variable $x$. We consider the first type boundary value problem for a one dimensional heat equation:

$$
\begin{aligned}
& L u=f(x, t) \quad \text { on } Q_{T}, \\
& u(x, 0)=u_{0}(x) \quad \text { on } \gamma_{2}, \\
& u(0, t)=u_{1}(t) \quad \text { on } \gamma_{1}, \quad u(b, t)=u_{2}(t) \quad \text { on } \gamma_{3},
\end{aligned}
$$

where $L \equiv \frac{\partial}{\partial t}-a \frac{\partial^{2}}{\partial x^{2}}$ and $a>0$ constant, then the functions on the right side of (5), (8) and (9) are

$$
\begin{aligned}
& u^{(0)}(x)=u_{0}(x) \\
& u^{(1)}(x)=a D_{x}^{2} u_{0}(x)+f(x, 0), \\
& u^{(q)}(x)=a \partial_{x}^{2} u^{(q-1)}(x)+f^{(q-1)}(x), \quad q=2,3, \ldots
\end{aligned}
$$

respectively, where $f^{(0)}(x)=f(x, 0)$ and $f^{(q)}(x)=\left.\partial_{t}^{q} f(x, t)\right|_{t=0}$. Also

$$
\begin{aligned}
& u_{1}^{(0)}(0)=\left.u_{1}(t)\right|_{t=0}, u_{2}^{(0)}(0)=\left.u_{2}(t)\right|_{t=0} \\
& u_{1}^{(q)}(0)=\left.D_{t}^{q} u_{1}(t)\right|_{t=0}, u_{2}^{(q)}(0)=\left.D_{t}^{q} u_{2}(t)\right|_{t=0}, \quad q=1,2, \ldots
\end{aligned}
$$


Furthermore, the conjugation conditions up to order $m \geq 0$ in (10) for the one dimensional problem (11)-(13) are derived as

$$
\begin{aligned}
& u^{(q)}(0)=u_{1}^{(q)}(0), \\
& u^{(q)}(b)=u_{2}^{(q)}(0),
\end{aligned}
$$

Problem 1 Let $\alpha \in(0,1)$

(i) The boundary value problem (11)-(13) satisfying the conditions

$$
\begin{aligned}
& u_{0}(x) \in C^{5+\alpha}(\bar{\Omega}), \quad f(x, t) \in C_{x, t}^{3+\alpha, \frac{3+\alpha}{2}}\left(\bar{Q}_{T}\right) \quad \text { and } \\
& u_{j}(t) \in C^{\frac{5+\alpha}{2}}\left(\bar{\sigma}_{T}\right), \quad j=1,2,
\end{aligned}
$$

and the conjugation conditions (19) up to second order $(q=0,1,2)$.

(ii) The boundary value problem (11)-(13) satisfying the conditions

$$
\begin{aligned}
& u_{0}(x) \in C^{7+\alpha}(\bar{\Omega}), \quad f(x, t) \in C_{x, t}^{5+\alpha, \frac{5+\alpha}{2}}\left(\bar{Q}_{T}\right) \quad \text { and } \\
& u_{j}(t) \in C^{\frac{7+\alpha}{2}}\left(\bar{\sigma}_{T}\right), \quad j=1,2,
\end{aligned}
$$

and the conjugation conditions (19) up to third order $(q=0,1,2,3)$.

Theorem 2 Problem 1(i) has a unique solution $u(x, t)$ belonging to the class $C_{x, t}^{5+\alpha, \frac{5+\alpha}{2}}\left(\bar{Q}_{T}\right)$. The Problem 1(ii) has a unique solution $u(x, t)$ belonging to the class $C_{x, t}^{7+\alpha, \frac{7+\alpha}{2}}\left(\bar{Q}_{T}\right)$.

Proof The proof of Theorem 2 follows from Theorem 1.

We define

$$
\begin{aligned}
& \bar{\omega}_{h}=\left\{x_{m}=m h, h=\frac{b}{N}, m=0, \ldots, N\right\}, \\
& \omega_{\tau}=\left\{t_{j}=j \tau, \tau=\frac{T}{M}, j=0, \ldots, M\right\},
\end{aligned}
$$

and $\bar{\omega}_{h, \tau}=\bar{\omega}_{h} \times \omega_{\tau}$ where the set of internal nodes are defined by

$$
\omega_{h, \tau}=\omega_{h} \times \omega_{\tau}=\left\{\left(x_{m}, t_{j}\right): m=1, \ldots, N-1, j=1, \ldots, M\right\} .
$$

The set of nodes on $\gamma_{i} i=1,2,3$ are presented by

$$
\begin{aligned}
& \omega_{0, \tau}=\left\{\left(0, t_{j}\right): t_{j}=j \tau, \tau=\frac{T}{M}, j=0, \ldots, M\right\}, \\
& \bar{\omega}_{h, 0}=\left\{\left(x_{m}, 0\right): x_{m}=m h, h=\frac{b}{N}, m=0, \ldots, N\right\}, \\
& \omega_{b, \tau}=\left\{\left(b, t_{j}\right): t_{j}=j \tau, \tau=\frac{T}{M}, j=0, \ldots, M\right\},
\end{aligned}
$$


respectively. Assume that $c_{1}, c_{2}, \ldots$ are positive constants independent from $h$ and $\tau$; in each section, those constants are enumerated anew. For the numerical solution of the Problem 1(i), we use the four point difference problem $(\varrho=3)$ and for the numerical solution of the Problem 1(ii), we use the six point symmetric difference problem $(\varrho=6)$ [9]. We denote the solution of these difference problems by $\widetilde{u}$ and use the notations $\widetilde{u}_{m}^{0}=\widetilde{u}\left(x_{m}, 0\right)$ on $\bar{\omega}_{h, 0}, \widetilde{u}_{0}^{j}=\widetilde{u}\left(0, t_{j}\right)$ on $\omega_{0, \tau}$, and $\widetilde{u}_{N}^{j}=\widetilde{u}\left(b, t_{j}\right)$ on $\omega_{b, \tau}$. The difference schemes are as follows:

$$
\begin{aligned}
& \widetilde{u}_{\bar{t}, m}^{h, \tau}=a \Theta^{\varrho} \widetilde{u}_{m}^{h, \tau}+\Phi f^{h, \tau} \quad \text { on } \omega_{h, \tau}, \quad \varrho=3 \quad \text { or } \quad \varrho=6, \\
& \widetilde{u}_{m}^{0}=u_{0}\left(x_{m}\right) \quad \text { on } \bar{\omega}_{h, 0}, \\
& \widetilde{u}_{0}^{j}=u_{1}\left(t_{j}\right) \quad \text { on } \omega_{0, \tau}, \quad \widetilde{u}_{N}^{j}=u_{2}\left(t_{j}\right) \quad \text { on } \omega_{b, \tau},
\end{aligned}
$$

where

$$
\begin{aligned}
& \widetilde{u}_{\bar{t}, m}^{h, \tau}=\frac{1}{\tau}\left(\widetilde{u}_{m}^{j+1}-\widetilde{u}_{m}^{j}\right), \\
& \Theta^{3} \widetilde{u}_{m}^{h, \tau}=\frac{1}{h^{2}}\left(\widetilde{u}_{m-1}^{j+1}-2 \widetilde{u}_{m}^{j+1}+\widetilde{u}_{m+1}^{j+1}\right), \\
& \Theta^{6} \widetilde{u}_{m}^{h, \tau}=\frac{1}{2 h^{2}}\left(\widetilde{u}_{m-1}^{j+1}-2 \widetilde{u}_{m}^{j+1}+\widetilde{u}_{m+1}^{j+1}\right)+\frac{1}{2 h^{2}}\left(\widetilde{u}_{m-1}^{j}-2 \widetilde{u}_{m}^{j}+\widetilde{u}_{m+1}^{j}\right), \\
& \Phi f^{h, \tau}= \begin{cases}f\left(x_{m}, t_{j+1}\right) & \text { if } \varrho=3, \\
\bar{f}=f\left(x_{m}, t_{j+\frac{1}{2}}\right) & \text { if } \varrho=6 .\end{cases}
\end{aligned}
$$

The operator $\Theta^{3} \widetilde{u}_{m}^{h, \tau}$ is the central difference formula and $\Theta^{6} \widetilde{u}_{m}^{h, \tau}$ is the averaging central difference formula with three points and six points respectively, for approximating $\partial_{x}^{2} u$. Here $t_{j+\frac{1}{2}}=t_{j}+0.5 \tau, f(x, t)$ is the given function in (11) and $u_{0}(x)$ given in (12), $u_{1}(t), u_{2}(t)$ given in (13) are the initial and boundary functions, respectively. Consider the following systems:

$$
\begin{aligned}
& \widehat{q}_{\bar{t}, \mathrm{~m}}^{h, \tau}=a \Theta^{\varrho} \widehat{q}_{m}^{h, \tau}+\widehat{g}^{h, \tau} \quad \text { on } \omega_{h, \tau}, \quad \varrho=3 \quad \text { or } \quad \varrho=6, \\
& \widehat{q}_{m}^{0}=0 \quad \text { on } \bar{\omega}_{h, 0}, \\
& \widehat{q}_{0}^{j}=0 \quad \text { on } \omega_{0, \tau}, \quad \widehat{q}_{N}^{j}=0 \quad \text { on } \omega_{b, \tau} \\
& \bar{q}_{\bar{t}, \mathrm{~h}}^{h, \tau}=a \Theta^{\varrho} \bar{q}_{m}^{h, \tau}+\bar{g}^{h, \tau} \quad \text { on } \omega_{h, \tau}, \quad \varrho=3 \quad \text { or } \quad \varrho=6, \\
& \bar{q}_{m}^{0} \geq 0 \quad \text { on } \bar{\omega}_{h, 0}, \\
& \bar{q}_{0}^{j} \geq 0 \quad \text { on } \omega_{0, \tau}, \quad \bar{q}_{N}^{j} \geq 0 \quad \text { on } \omega_{b, \tau}
\end{aligned}
$$

where $\widehat{g}^{h, \tau}, \bar{g}^{h, \tau}$ are given functions and $\left|\widehat{g}^{h, \tau}\right| \leq \bar{g}^{h, \tau}$ on $\omega_{h, \tau}$ also $\widehat{q}_{\bar{t}, m}^{h, \tau}, \bar{q}_{\bar{t}, m}^{h, \tau}$ are difference formulae analogous to (31) and $\Theta^{\varrho} \widehat{q}_{m}^{h, \tau}, \Theta^{\varrho} \bar{q}_{m}^{h, \tau}$ are difference formulae analogous to (32) or (33) for $\varrho=3$ or $\varrho=6$, respectively.

Lemma 3 The solution $\widehat{q}$ of the system (35)-(37) and the solution $\bar{q}$ of the system (38)-(40) satisfy the inequality

$$
|\widehat{q}| \leq \bar{q} \quad \text { on } \bar{\omega}_{h, \tau}
$$


for any $r$ by the four point implicit scheme $(\varrho=3)$ and for $r \leq 1$, by the six point symmetric implicit scheme $(\varrho=6)$ where $r=\frac{a \tau}{h^{2}}$.

Proof Taking into consideration that the canonical form of the equation $\widehat{q}_{\bar{t}, m}^{h, \tau}=a \Theta^{3} \widehat{q}_{m}^{h, \tau}+$ $\widehat{g}^{h, \tau}$ is

$$
\left(\frac{1}{\tau}+\frac{2 a}{h^{2}}\right) \widehat{q}_{m}^{j+1}=\frac{a}{h^{2}}\left(\widehat{q}_{m-1}^{j+1}+\widehat{q}_{m+1}^{j+1}\right)+\frac{1}{\tau} \widehat{q}_{m}^{j}+\widehat{g}^{h, \tau}
$$

in the form $A(P) \widehat{q}(P)=\sum_{Q \in \operatorname{Patt}(P)} B(P, Q) \widehat{q}(Q)+F(P)$ where $P=P\left(x_{m}, t_{j+1}\right)$ as a node of the grid $\omega_{h, \tau}$ and $\operatorname{Patt}(P)$ consists of the nodes $Q_{1}=\left(x_{m}, t_{j}\right), Q_{2}=\left(x_{m-1}, t_{j+1}\right), Q_{3}=$ $\left(x_{m+1}, t_{j+1}\right) \in \bar{\omega}_{h, \tau}$. It is easily seen that $A(P)>0, B(P, Q)>0$ for every $Q \in \operatorname{Patt}(P)$ and $D(P)=0$ where $D(P)=A(P)-\sum_{Q \in \operatorname{Patt}(P)} B(P, Q)$. Similarly the canonical form of the equation $\widehat{q}_{\bar{t}, m}^{h, \tau}=a \Theta^{6} \widehat{q}_{m}^{h, \tau}+\widehat{g}^{h, \tau}$ is

$$
\left(\frac{1}{\tau}+\frac{a}{h^{2}}\right) \widehat{q}_{m}^{j+1}=\frac{a}{2 h^{2}}\left(\widehat{q}_{m-1}^{j+1}+\widehat{q}_{m+1}^{j+1}\right)+\frac{a}{2 h^{2}}\left(\widehat{q}_{m-1}^{j}+\widehat{q}_{m+1}^{j}\right)+\left(\frac{1}{\tau}-\frac{a}{h^{2}}\right) \widehat{q}_{m}^{j}+\widehat{g}^{h, \tau}
$$

where $P=P\left(x_{m}, t_{j+1}\right)$ and $\operatorname{Patt}(P)$ consists of the nodes $Q_{1}=\left(x_{m}, t_{j}\right), Q_{2}=\left(x_{m-1}, t_{j+1}\right)$, $Q_{3}=\left(x_{m+1}, t_{j+1}\right), Q_{4}=\left(x_{m-1}, t_{j}\right), Q_{5}=\left(x_{m+1}, t_{j}\right)$. Here $A(P)>0, D(P)=0$ and $B(P, Q) \geq 0$ for every $Q \in \operatorname{Patt}(P)$ if $r=\frac{a \tau}{h^{2}} \leq 1$. The proof follows from the comparison theorem (see Chap. 4 in [9]) because the coefficients of the finite difference schemes (42) and (43) satisfy all conditions of the comparison theorem for any $r$ and for $r \leq 1$, respectively.

Lemma 4 For the solution of the problem

$$
\begin{aligned}
& \widehat{q}_{\bar{t}, m}^{h, \tau}=a \Theta^{\varrho} \widehat{q}_{m}^{h, \tau}+\beta \quad \text { on } \omega_{h, \tau}, \quad \varrho=3 \quad \text { or } \quad \varrho=6, \\
& \widehat{q}_{m}^{0}=0 \quad \text { on } \bar{\omega}_{h, 0}, \\
& \widehat{q}_{0}^{j}=0 \quad \text { on } \omega_{0, \tau}, \quad \widehat{q}_{N}^{j}=0 \quad \text { on } \omega_{b, \tau},
\end{aligned}
$$

the following inequality holds true:

$$
\widehat{q} \leq \Upsilon d \beta \quad \text { on } \bar{\omega}_{h, \tau}
$$

where

$$
\begin{aligned}
& \beta=\beta(h, \tau)= \begin{cases}h^{2}+\tau & \text { for } \varrho=3, \\
h^{2}+\tau^{2} & \text { for } \varrho=6,\end{cases} \\
& d=\max \left[\frac{b}{2 a}, 1\right],
\end{aligned}
$$

for any $r$ by the four point implicit scheme $(\varrho=3)$ and for $r \leq 1$, by the symmetric six point implicit scheme $(\varrho=6)$. Here, $\Upsilon=\Upsilon(x, t)$ is the distance from the current point $(x, t) \in \bar{\omega}_{h, \tau}$ to the boundary $\gamma$ of $Q_{T}$. 
Proof For the four point implicit scheme ( $\varrho=3)$, we consider the functions

$$
\bar{q}_{1}^{3}(x, t)=\frac{1}{2}\left(h^{2}+\tau\right)\left(\frac{b x-x^{2}}{a}\right) \geq 0, \quad \bar{q}_{2}^{3}(x, t)=\left(h^{2}+\tau\right) t \geq 0 \quad \text { on } \bar{\omega}_{h, \tau}
$$

which are the solutions of $q_{\bar{t}, m}^{h, \tau}=a \Theta^{3} q_{m}^{h, \tau}+h^{2}+\tau$ on $\omega_{h, \tau}$. On the basis of Lemma 3 we obtain

$$
\widehat{q} \leq \min _{i=1,2} \bar{q}_{i}^{3}(x, t) \leq \Upsilon d\left(h^{2}+\tau\right) \quad \text { on } \bar{\omega}_{h, \tau} .
$$

For the six point symmetric implicit scheme $(\varrho=6)$, we consider the functions

$$
\bar{q}_{1}^{6}(x, t)=\frac{1}{2}\left(h^{2}+\tau^{2}\right)\left(\frac{b x-x^{2}}{a}\right) \geq 0, \quad \bar{q}_{2}^{6}(x, t)=\left(h^{2}+\tau^{2}\right) t \geq 0 \quad \text { on } \bar{\omega}_{h, \tau}
$$

which are the solutions of $q_{\bar{t}, m}^{h, \tau}=a \Theta^{6} q_{m}^{h, \tau}+h^{2}+\tau^{2}$ on $\omega_{h, \tau}$. Using Lemma 3 we obtain

$$
\widehat{q} \leq \min _{i=1,2} \bar{q}_{i}^{6}(x, t) \leq \Upsilon d\left(h^{2}+\tau^{2}\right) \quad \text { on } \bar{\omega}_{h, \tau} .
$$

Theorem 5 The solution $\tilde{u}$ of the four point finite difference problem $(28)-(30)(\varrho=3)$ satisfies the following pointwise estimation:

$$
|\tilde{u}-u| \leq c_{1} \Upsilon\left(h^{2}+\tau\right),
$$

for any value of $r$ where $u$ is the exact solution of Problem 1(i). The solution $\tilde{u}$ of the six point finite difference problem (28)-(30) $(\varrho=6)$ satisfies the following pointwise estimation:

$$
|\widetilde{u}-u| \leq c_{2} \Upsilon\left(h^{2}+\tau^{2}\right),
$$

for $r \leq 1$ where $u$ is the exact solution of Problem 1(ii).

Proof On the basis of Theorem 2, the exact solution $u$ of Problem 1(i) belongs to $C_{x, t}^{5+\alpha, \frac{5+\alpha}{2}}\left(\bar{Q}_{T}\right)$. Therefore, $\partial_{x}^{4} u$ and $\partial_{t}^{2} u$ are bounded up to the boundary. Let $\varepsilon_{u}^{h, \tau}=\widetilde{u}-u$ on $\bar{\omega}_{h, \tau}$. Obviously the error function $\varepsilon_{u}^{h, \tau}$ satisfies

$$
\begin{array}{ll}
\varepsilon_{u, \bar{t}, m}^{h, \tau}=a \Theta^{3} \varepsilon_{u, m}^{h, \tau}+\psi_{u} & \text { on } \omega_{h, \tau}, \\
\varepsilon_{u, m}^{0}=0 \quad \text { on } \bar{\omega}_{h, 0}, & \\
\varepsilon_{u, 0}^{j}=0 & \text { on } \omega_{0, \tau}, \quad \varepsilon_{u, N}^{j}=0 \quad \text { on } \omega_{b, \tau},
\end{array}
$$

where $\psi_{u}=a \Theta^{3} u-u_{\bar{t}, m}+\Phi f^{h, \tau}$. Using Taylor's formula for the function $u(x, t)$ about the node $\left(x_{m}, t_{j+1}\right)$ shows that $\psi_{u}=O\left(h^{2}+\tau\right)$ and applying Lemma 3 to the problem (44)(46) for $\varrho=3$ and (56)-(58) and on the basis of Lemma 4 we obtain $\left|\varepsilon_{u}^{h, \tau}\right| \leq c_{1} \Upsilon\left(h^{2}+\tau\right)$. From Theorem 2, the exact solution $u$ of Problem 1(ii) belongs to $C_{x, t}^{7+\frac{7+\alpha}{2}}\left(\bar{Q}_{T}\right)$. Hence, 
the derivatives $\partial_{x}^{4} u, \partial_{t}^{3} u$ are bounded up to the boundary. The error function $\varepsilon_{u}^{h, \tau}$ satisfies the following difference problem:

$$
\begin{aligned}
& \varepsilon_{u, \bar{t}, m}^{h, \tau}=a \Theta^{6} \varepsilon_{u, m}^{h, \tau}+\psi_{u} \quad \text { on } \omega_{h, \tau}, \\
& \varepsilon_{u, m}^{0}=0 \quad \text { on } \bar{\omega}_{h, 0} \text {, } \\
& \varepsilon_{u, 0}^{j}=0 \quad \text { on } \omega_{0, \tau}, \quad \varepsilon_{u, N}^{j}=0 \quad \text { on } \omega_{b, \tau} \text {. }
\end{aligned}
$$

Using Taylor's formula for the function $u(x, t)$ about the node $\left(x_{m}, t_{j+\frac{1}{2}}\right)$ shows that $\psi_{u}=$ $O\left(h^{2}+\tau^{2}\right)$. Applying Lemma 3 to the six point implicit difference problem (44)-(46) $(\varrho=6)$ and (59)-(61) and on the basis of Lemma 4 we obtain $\left|\varepsilon_{u}^{h, \tau}\right| \leq c_{2} \Upsilon\left(h^{2}+\tau^{2}\right)$.

\section{Implicit four point difference approximation of $\partial_{x} u$}

\section{Problem 2}

(i) Given the Problem 1(i), we denote $p_{i}=\partial_{x} u$ on $\gamma_{i}, i=1,2,3$ and set up the next boundary value problem for $v=\partial_{x} u$,

$$
\begin{aligned}
& L v=\partial_{x} f(x, t) \quad \text { on } Q_{T}, \\
& v(x, 0)=p_{2} \quad \text { on } \gamma_{2}, \\
& v(0, t)=p_{1} \quad \text { on } \gamma_{1}, \quad v(b, t)=p_{3} \quad \text { on } \gamma_{3},
\end{aligned}
$$

where $f(x, t)$ is the given function in (11).

We take

$$
\begin{aligned}
& p_{1 h}=\frac{1}{2 h}\left(-3 u_{1}(t)+4 \widetilde{u}(h, t)-\widetilde{u}(2 h, t)\right) \quad \text { on } \omega_{0, \tau}, \\
& p_{2 h}=\partial_{x} u_{0}(x) \text { on } \bar{\omega}_{h, 0}, \\
& p_{3 h}=\frac{1}{2 h}\left(3 u_{2}(t)-4 \widetilde{u}(b-h, t)+\widetilde{u}(b-2 h, t)\right) \text { on } \omega_{b, \tau},
\end{aligned}
$$

and $u_{0}(x)$ given in (12), $u_{1}(t), u_{2}(t)$ given in (13) are the initial and boundary functions, respectively, $\widetilde{u}$ is the solution of the four point difference problem $(28)-(30)(\varrho=3)$.

Lemma 6 The following inequality holds:

$$
\left|p_{i h}(\widetilde{u})-p_{i h}(u)\right| \leq c_{1}\left(h^{2}+\tau\right), \quad i=1,3,
$$

where $u$ is the solution of the differential Problem 1(i) and $\widetilde{u}$ is the solution of the four point difference problem (28)-(30) $(\varrho=3)$.

Proof Taking into consideration Theorem 2, and using (65) and (67) and Theorem 5, we have

$$
\left|p_{i h}(\widetilde{u})-p_{i h}(u)\right| \leq \frac{1}{2 h}\left(4\left(c_{2} h\right)\left(h^{2}+\tau\right)+\left(c_{2} 2 h\right)\left(h^{2}+\tau\right)\right) \leq c_{1}\left(h^{2}+\tau\right), \quad i=1,3 .
$$


Lemma 7 The following inequality is true:

$$
\max _{\omega_{0, \tau} \cup \omega_{b, \tau}}\left|p_{i h}(\widetilde{u})-p_{i}\right| \leq c_{3}\left(h^{2}+\tau\right), \quad i=1,3,
$$

where $\widetilde{u}$ is the solution of the four point difference problem $(28)-(30)(\varrho=3)$.

Proof On the basis of Theorem 2, the exact solution $u \in C_{x, t}^{5+\alpha, \frac{5+\alpha}{2}}\left(\bar{Q}_{T}\right)$. Then at the end points $(0, \sigma \tau) \in \omega_{0, \tau}$ and $(b, \sigma \tau) \in \omega_{b, \tau}$ of each line segment $[(x, t): 0 \leq x \leq b, 0<t \leq T]$ (65) and (67) give the second order approximation of $\partial_{x} u$, respectively. From the truncation error formula (see [11]) it follows that

$$
\max _{\omega_{0, \tau} \cup \omega_{b, \tau}}\left|p_{i h}(u)-p_{i}\right| \leq \frac{h^{2}}{3} \max _{\bar{Q}_{T}}\left|\partial_{x}^{3} u\right| \leq c_{3} h^{2}, \quad i=1,3 .
$$

Using Lemma 6 and the estimation (66), (71) follows.

We construct the following difference problem for the numerical solution of Problem 2(i):

$$
\begin{aligned}
& \widetilde{v}_{\bar{t}, m}^{h, \tau}=a \Theta^{3} \widetilde{v}_{m}^{h, \tau}+\Phi \partial_{x} f^{h, \tau} \quad \text { on } \omega_{h, \tau}, \\
& \widetilde{v}_{m}^{0}=p_{2 h} \quad \text { on } \bar{\omega}_{h, 0}, \\
& \widetilde{v}_{0}^{j}=p_{1 h}(\widetilde{u}) \quad \text { on } \omega_{0, \tau}, \quad \widetilde{v}_{N}^{j}=p_{3 h}(\widetilde{u}) \quad \text { on } \omega_{b, \tau},
\end{aligned}
$$

where the $p_{\text {ih }}$ are defined by (65)-(67) and $\Phi \partial_{x} f^{h, \tau}=\left.\partial_{x} f\right|_{\left(x_{m}, t_{j+1}\right)}$ and $\widetilde{u}$ is the solution of the four point difference problem $(28)-(30)(\varrho=3)$.

Theorem 8 The solution $\widetilde{v}$ of the finite difference problem (72)-(74) satisfies

$$
\max _{\bar{\omega}_{h, \tau}}|\widetilde{v}-v| \leq c_{4}\left(h^{2}+\tau\right)
$$

where $v=\partial_{x} u$ is the exact solution of Problem 2(i).

Proof Let

$$
\varepsilon_{v}^{h, \tau}=\widetilde{v}-v \quad \text { on } \bar{\omega}_{h, \tau}
$$

where $v=\partial_{x} u$. Denote by $\left\|\varepsilon_{v}^{h, \tau}\right\|=\max _{\bar{\omega}_{h, \tau}} \widetilde{v}-v \mid$. From (72)-(74) and (76) we have

$$
\begin{aligned}
& \varepsilon_{v, \bar{t}, m}^{h, \tau}=a \Theta^{3} \varepsilon_{v, m}^{h, \tau}+\psi_{v} \quad \text { on } \omega_{h, \tau}, \\
& \varepsilon_{v, m}^{0}=0 \quad \text { on } \bar{\omega}_{h, 0}, \\
& \varepsilon_{v, 0}^{j}=p_{1 h}(\widetilde{u})-v \quad \text { on } \omega_{0, \tau}, \quad \varepsilon_{v, N}^{j}=p_{3 h}(\widetilde{u})-v \quad \text { on } \omega_{b, \tau},
\end{aligned}
$$

where $\psi_{v}=a \Theta^{3} v-v_{t, m}+\Phi \partial_{x} f^{h, \tau}$. We take

$$
\varepsilon_{v}^{h, \tau}=\varepsilon_{v}^{1, h, \tau}+\varepsilon_{v}^{2, h, \tau}
$$


where

$$
\begin{aligned}
& \varepsilon_{v, \bar{t}, m}^{1, h, \tau}=a \Theta^{3} \varepsilon_{v, m}^{1, h, \tau} \text { on } \omega_{h, \tau}, \\
& \varepsilon_{v, m}^{1,0}=0 \quad \text { on } \bar{\omega}_{h, 0}, \\
& \varepsilon_{v, 0}^{1, j}=p_{1 h}(\widetilde{u})-v \quad \text { on } \omega_{0, \tau}, \quad \varepsilon_{v, N}^{1, j}=p_{3 h}(\widetilde{u})-v \quad \text { on } \omega_{b, \tau}, \\
& \varepsilon_{v, \bar{t}, m}^{2, h, \tau}=a \Theta^{3} \varepsilon_{v, m}^{2, h, \tau}+\psi_{v} \quad \text { on } \omega_{h, \tau}, \\
& \varepsilon_{v, m}^{2,0}=0 \quad \text { on } \bar{\omega}_{h, 0}, \\
& \varepsilon_{v, 0}^{2, j}=0 \quad \text { on } \omega_{0, \tau}, \quad \varepsilon_{v, N}^{2, j}=0 \quad \text { on } \omega_{b, \tau} .
\end{aligned}
$$

From Lemma 7 and by maximum principle for the solution of the system (81)-(83) we have

$$
\max _{\bar{\omega}_{h, \tau}}\left|\varepsilon_{v}^{1, h, \tau}\right| \leq \max _{i=1,3} \max _{\bar{\omega}_{h, \tau}}\left|p_{i h}(\widetilde{u})-v\right| \leq c_{4}\left(h^{2}+\tau\right)
$$

The solution $\varepsilon_{v}^{2, h, \tau}$ of system (84)-(86) is the error of the approximate solution obtained by the finite difference method for the boundary value Problem 2(i) when the boundary values satisfy the conditions

$$
\begin{aligned}
& p_{2} \in C^{4+\alpha}(\bar{\Omega}), \quad \partial_{x} f(x, t) \in C_{x, t}^{2+\alpha, 1+\frac{\alpha}{2}}\left(\bar{Q}_{T}\right), \quad p_{i} \in C^{2+\frac{\alpha}{2}}\left(\bar{\sigma}_{T}\right), \quad i=1,3, \\
& \left\{\begin{array}{l}
p_{1}^{(q)}(0)=v^{(q)}(0), \\
p_{3}^{(q)}(0)=v^{(q)}(b),
\end{array}\right.
\end{aligned}
$$

Since the function $v=\partial_{x} u$ satisfies Eq. (62) with the initial function $p_{2}$ on $\gamma_{2}$ and boundary functions $p_{1}, p_{3}$ on $\gamma_{1}$ and $\gamma_{3}$, respectively, and on the basis of Theorem 1 and the maximum principle, we obtain

$$
\max _{\bar{\omega}_{h, \tau}}\left|\varepsilon_{v}^{2, h, \tau}\right| \leq c_{5}\left(h^{2}+\tau\right),
$$

and using (80), (87) and (90) we obtain (75).

\section{Implicit six point symmetric difference approximation of $\partial_{x} u$}

\section{Problem 2}

(ii) Given the Problem 1(ii), we denote $p_{i}=\partial_{x} u$ on $\gamma_{i}, i=1,2,3$ and set up the boundary value problem (62)-(64) for $v=\partial_{x} u$.

Lemma 9 The following inequality holds:

$$
\left|p_{i h}(\widetilde{u})-p_{i h}(u)\right| \leq c_{1}\left(h^{2}+\tau^{2}\right), \quad i=1,3,
$$

where $u$ is the solution of the differential Problem 1(ii) and $\widetilde{u}$ is the solution of the symmetric six point difference problem (28)-(30) ( $\varrho=6)$ for $r \leq 1$ and $p_{\text {ih }}$ are defined by (65)-(67). 
Proof On the basis of Theorem 2, and from (65), (67) and using Theorem 5, we have

$$
\begin{aligned}
\left|p_{i h}(\widetilde{u})-p_{i h}(u)\right| & \leq \frac{1}{2 h}\left(4\left(c_{2} h\right)\left(h^{2}+\tau^{2}\right)+\left(c_{2} 2 h\right)\left(h^{2}+\tau^{2}\right)\right) \\
& \leq c_{1}\left(h^{2}+\tau^{2}\right), \quad i=1,3 .
\end{aligned}
$$

Lemma 10 The following inequality is true:

$$
\max _{\omega_{0, \tau} \cup \omega_{b, \tau}}\left|p_{i h}(\widetilde{u})-p_{i}\right| \leq c_{3}\left(h^{2}+\tau^{2}\right), \quad i=1,3,
$$

where $\widetilde{u}$ is the solution of the six point difference problem (28)-(30) $(\varrho=6)$ for $r \leq 1$.

Proof Using Theorem 2, the proof is analogous to the proof of Lemma 7.

We propose the following six point difference problem for the numerical solution of Problem 2(ii):

$$
\begin{aligned}
& \widetilde{v}_{\bar{t}, m}^{h, \tau}=a \Theta^{6} \widetilde{v}_{m}^{h, \tau}+\Phi \partial_{x} f^{h, \tau} \quad \text { on } \omega_{h, \tau}, \\
& \widetilde{v}_{m}^{0}=p_{2 h} \quad \text { on } \bar{\omega}_{h, 0}, \\
& \widetilde{v}_{0}^{j}=p_{1 h}(\widetilde{u}) \quad \text { on } \omega_{0, \tau}, \quad \widetilde{v}_{N}^{j}=p_{3 h}(\widetilde{u}) \quad \text { on } \omega_{b, \tau},
\end{aligned}
$$

where $p_{i h}$ are defined by (65)-(67) and $\Phi \partial_{x} f^{h, \tau}=\left.\partial_{x} f\right|_{\left(x_{m}, t_{j+\frac{1}{2}}\right)}$ and $\tilde{u}$ is the solution of the six point difference problem (28)-(30) $(\varrho=6)$ for $r \leq 1$.

Theorem 11 For $r \leq 1$, the solution $\widetilde{v}$ of the finite difference problem (94)-(96) satisfies

$$
\max _{\bar{\omega}_{h, \tau}}|\widetilde{v}-v| \leq c_{4}\left(h^{2}+\tau^{2}\right),
$$

where $v=\partial_{x} u$ is the exact solution of Problem 2(ii).

Proof The proof is analogous to the proof of Theorem 8. From (94)-(96) and (76) we have

$$
\begin{aligned}
& \varepsilon_{v, \bar{t}, m}^{h, \tau}=a \Theta^{6} \varepsilon_{v, m}^{h, \tau}+\psi_{v} \quad \text { on } \omega_{h, \tau}, \\
& \varepsilon_{v, m}^{0}=0 \quad \text { on } \bar{\omega}_{h, 0}, \\
& \varepsilon_{v, 0}^{j}=p_{1 h}(\widetilde{u})-v \quad \text { on } \omega_{0, \tau}, \quad \varepsilon_{v, N}^{j}=p_{3 h}(\widetilde{u})-v \quad \text { on } \omega_{b, \tau},
\end{aligned}
$$

where $\psi_{v}=a \Theta^{6} v-v_{\bar{t}, m}+\Phi \partial_{x} f^{h, \tau}$. We take

$$
\varepsilon_{v}^{h, \tau}=\varepsilon_{v}^{1, h, \tau}+\varepsilon_{v}^{2, h, \tau},
$$

where

$$
\varepsilon_{v, \bar{t}, m}^{1, h, \tau}=a \Theta^{6} \varepsilon_{v, m}^{1, h, \tau} \quad \text { on } \omega_{h, \tau},
$$




$$
\begin{aligned}
& \varepsilon_{v, m}^{1,0}=0 \quad \text { on } \bar{\omega}_{h, 0}, \\
& \varepsilon_{v, 0}^{1, j}=p_{1 h}(\widetilde{u})-v \quad \text { on } \omega_{0, \tau}, \quad \varepsilon_{v, N}^{1, j}=p_{3 h}(\widetilde{u})-v \quad \text { on } \omega_{b, \tau}, \\
& \varepsilon_{v, \bar{t}, m}^{2, h, \tau}=a \Theta^{6} \varepsilon_{v, m}^{2, h, \tau}+\psi_{v} \quad \text { on } \omega_{h, \tau}, \\
& \varepsilon_{v, m}^{2,0}=0 \quad \text { on } \bar{\omega}_{h, 0}, \\
& \varepsilon_{v, 0}^{2, j}=0 \quad \text { on } \omega_{0, \tau}, \quad \varepsilon_{v, N}^{2, j}=0 \quad \text { on } \omega_{b, \tau} .
\end{aligned}
$$

From Lemma 10 and by the maximum principle for the solution of the system (102)-(104) we have

$$
\max _{\bar{\omega}_{h, \tau}}\left|\varepsilon_{v}^{1, h, \tau}\right| \leq \max _{i=1,3} \max _{\bar{\omega}_{h, \tau}}\left|p_{i h}(\widetilde{u})-v\right| \leq c_{4}\left(h^{2}+\tau^{2}\right)
$$

The solution $\varepsilon_{v}^{2, h, \tau}$ of system (105)-(107) is the error of the approximate solution obtained by the finite difference method for the boundary value Problem 2(ii) when the boundary values satisfy the conditions

$$
\begin{aligned}
& p_{2} \in C^{6+\alpha}(\bar{\Omega}), \quad \partial_{x} f(x, t) \in C_{x, t}^{4+\alpha, 2+\frac{\alpha}{2}}\left(\bar{Q}_{T}\right), \quad p_{i} \in C^{3+\frac{\alpha}{2}}\left(\bar{\sigma}_{T}\right), i=1,3, \\
& \left\{\begin{array}{l}
p_{1}^{(q)}(0)=v^{(q)}(0), \\
p_{3}^{(q)}(0)=v^{(q)}(b),
\end{array}\right.
\end{aligned}
$$

Since the function $v=\partial_{x} u$ satisfies Eq. (62) with the initial function $p_{2}$ on $\gamma_{2}$ and boundary functions $p_{1}, p_{3}$ on $\gamma_{1}$ and $\gamma_{3}$, respectively and on the basis of Theorem 1 and the maximum principle in Chap. 4 of [9] we obtain

$$
\max _{\bar{\omega}_{h, \tau}}\left|\varepsilon_{v}^{2, h, \tau}\right| \leq c_{5}\left(h^{2}+\tau^{2}\right)
$$

using (80), (108) and (111) we obtain (97).

\section{Numerical aspects}

Two problems are considered such that the first type boundary value problem for the one dimensional heat equation in Example 1 and in Example 2 are chosen as examples for Problem 1(i) and Problem 1(ii), respectively, and the exact first derivatives of their solutions with respect to $x$ are known. The third example is also given as an example of Problem 1(ii), however, the exact first derivative of the solution of this problem with respect to $x$ is not given. All the computations are carried out in double precision using the Fortran programming language. For the constructed examples we take $Q_{T}=\{(x, t): 0<x<1,0<t \leq 1\}, \gamma_{1}=\{(0, t): 0 \leq t \leq 1\}, \gamma_{2}=\{(x, 0): 0 \leq x \leq 1\}$, and $\gamma_{3}=\{(1, t): 0 \leq t \leq 1\}$, and the constant $a$ in the operator $L \equiv \frac{\partial}{\partial t}-a \frac{\partial^{2}}{\partial x^{2}}$ is taken as $a=1$.

\section{Example 1}

$$
\begin{aligned}
& L u=f(x, t) \quad \text { on } Q_{T}, \\
& u(x, 0)=x^{\frac{26}{5}}+\sin \left(\frac{\pi}{2} x\right) \text { on } \gamma_{2},
\end{aligned}
$$




$$
\begin{aligned}
& u(0, t)=t^{\frac{13}{5}} \text { on } \gamma_{1}, \\
& u(1, t)=t^{\frac{13}{5}}+\cos \left(t^{\frac{13}{5}}\right)+1 \text { on } \gamma_{3},
\end{aligned}
$$

where $f(x, t)=-\frac{13}{5} x^{\frac{26}{5}} t^{\frac{8}{5}} \sin \left(t^{\frac{13}{5}}\right)+\frac{13}{5} t^{\frac{8}{5}}-\frac{26}{5} \frac{21}{5} x^{\frac{16}{5}} \cos \left(t^{\frac{13}{5}}\right)+\frac{\pi^{2}}{4} \sin \left(\frac{\pi}{2} x\right)$. Using the proposed implicit four point difference scheme (28)-(30) $(\varrho=3)$ we obtain the approximate solution $\tilde{u}$ by the applying the Gauss-Thomas method [12] for solving an algebraic system of equations at each time level with space step size $h=2^{-\mu}$ and time step size $\tau=2^{-\lambda}$ where $\mu, \lambda$ are positive integers. Next the boundary value problem for $v=\frac{\partial u}{\partial x}$ is constructed using the obtained approximate solution $\tilde{u}$ and the proposed Problem 2(i). Furthermore, the approximate solution $\widetilde{v}$ of the difference problem (72)-(74) is obtained at the same grid points. The exact solution is known: $v(x, t)=\frac{26}{5} x^{\frac{21}{5}} \cos \left(t^{\frac{13}{5}}\right)+\frac{\pi}{2} \cos \left(\frac{\pi}{2} x\right)$; and we denote the maximum error on the grid points by $\left\|\varepsilon_{v}^{h, \tau}\right\|=\max _{\bar{\omega}_{h, \tau}}|\widetilde{v}-v|$. Table 1 demonstrates the maximum errors for fixed $r=2^{-\omega}, \omega=2,3$, and the order of convergence

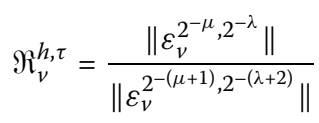

of $\widetilde{v}$ with respect to $h$ and $\tau$ for Example 1 . Table 2 represents the maximum errors for $h=2^{-9}, \tau=2^{-\lambda}, \lambda=6,7,8,9,10,11$ and the order of convergence

$$
\Re_{v}^{\tau}=\frac{\left\|\varepsilon_{v}^{h, 2^{-\lambda}}\right\|}{\left\|\varepsilon_{v}^{h, 2^{-(\lambda+1)}}\right\|}
$$

of $\widetilde{v}$ with respect to $\tau$ for Example 1. According to the definition of the maximum error the third and fourth columns of Table 1 and Table 2 present the theoretical upper bound errors given in (75). Note that the $O\left(h^{2}+\tau\right)$ order of convergence corresponds to $\asymp 2^{2}$ of the quantities defined by (113), and $\asymp 2^{1}$ of the quantities defined by (114). Figure 1 presents the error function $\left|\varepsilon_{v}^{2^{-7}, 2^{-17}}\right|=|\widetilde{v}-v|$ for $h=2^{-7}$, and $\tau=2^{-17}$ for Example 1. The maximum errors $\left\|\varepsilon_{v}^{2^{-9}, \tau}\right\|$ when $h=2^{-9}$, with respect to $\tau$, are shown in Fig. 2 and the maximum errors $\left\|\varepsilon_{v}^{h, 2^{-17}}\right\|$ when $\tau=2^{-17}$, with respect to $h$, are demonstrated by Fig. 3 for Example 1 . Figure 4 shows the exact solution $v(x, t)=\partial_{x} u$, and the grid function $v^{2^{-7}, 2^{-17}}$ presenting the approximate solution $\widetilde{v}$ of $\partial_{x} u$ when $h=2^{-7}, \tau=2^{-17}$ for Example 1 is presented in Fig. 5.

Example 2

$$
\begin{aligned}
& L u=f(x, t) \quad \text { on } Q_{T}, \\
& u(x, 0)=\frac{5}{36} \frac{5}{18} x^{\frac{36}{5}}+\sin \left(\frac{\pi x}{2}\right) \quad \text { on } \gamma_{2},
\end{aligned}
$$

Table 1 Maximum errors and the order of convergence of approximate solution $\tilde{v}$ with respect to $h$ and $\tau$ for Example 1

\begin{tabular}{lllll}
\hline$\left(h=2^{-\mu}, \tau=2^{-\lambda}\right)$ & $\left(h=2^{-(\mu+1)}, \tau=2^{-(\lambda+2)}\right)$ & $\left\|\varepsilon_{v}^{2^{-\mu}, 2^{-\lambda}}\right\|$ & $\left\|\varepsilon_{v}^{2^{-(\mu+1)}, 2^{-(\lambda+2)}}\right\|$ & $\Re_{v}^{h, r}$ \\
\hline$\left(2^{-5}, 2^{-12}\right)$ & $\left(2^{-6}, 2^{-14}\right)$ & $2.438 \mathrm{E}-2$ & $6.383 \mathrm{E}-3$ & 3.820 \\
$\left(2^{-6}, 2^{-14}\right)$ & $\left(2^{-7}, 2^{-16}\right)$ & $6.383 \mathrm{E}-3$ & $1.633 \mathrm{E}-3$ & 3.909 \\
$\left(2^{-5}, 2^{-13}\right)$ & $\left(2^{-6}, 2^{-15}\right)$ & $2.439 \mathrm{E}-2$ & $6.385 \mathrm{E}-3$ & 3.820 \\
$\left(2^{-6}, 2^{-15}\right)$ & $\left(2^{-7}, 2^{-17}\right)$ & $6.385 \mathrm{E}-3$ & $1.633 \mathrm{E}-3$ & 3.910 \\
\hline
\end{tabular}


Table 2 Maximum errors and the order of convergence of approximate solution $\tilde{v}$ with respect to $\tau$ for Example 1

\begin{tabular}{lllll}
\hline$\left(h=2^{-\mu}, \tau=2^{-\lambda}\right)$ & $\left(h=2^{-\mu}, \tau=2^{-(\lambda+1)}\right)$ & $\left\|\varepsilon_{V}^{2^{-\mu}, 2^{-\lambda}}\right\|$ & $\left\|\varepsilon_{v}^{2^{-\mu}, 2^{-(\lambda+1)}}\right\|$ & $\Re_{v}^{\tau}$ \\
\hline$\left(2^{-9}, 2^{-6}\right)$ & $\left(2^{-9}, 2^{-7}\right)$ & $6.087 \mathrm{E}-2$ & $3.058 \mathrm{E}-2$ & 1.991 \\
$\left(2^{-9}, 2^{-7}\right)$ & $\left(2^{-9}, 2^{-8}\right)$ & $3.058 \mathrm{E}-2$ & $1.531 \mathrm{E}-2$ & 1.997 \\
$\left(2^{-9}, 2^{-8}\right)$ & $\left(2^{-9}, 2^{-9}\right)$ & $1.531 \mathrm{E}-2$ & $7.634 \mathrm{E}-3$ & 2.006 \\
$\left(2^{-9}, 2^{-9}\right)$ & $\left(2^{-9}, 2^{-10}\right)$ & $7.634 \mathrm{E}-3$ & $3.791 \mathrm{E}-3$ & 2.014 \\
$\left(2^{-9}, 2^{-10}\right)$ & $\left(2^{-9}, 2^{-11}\right)$ & $3.791 \mathrm{E}-3$ & $1.867 \mathrm{E}-3$ & 2.031 \\
\hline
\end{tabular}

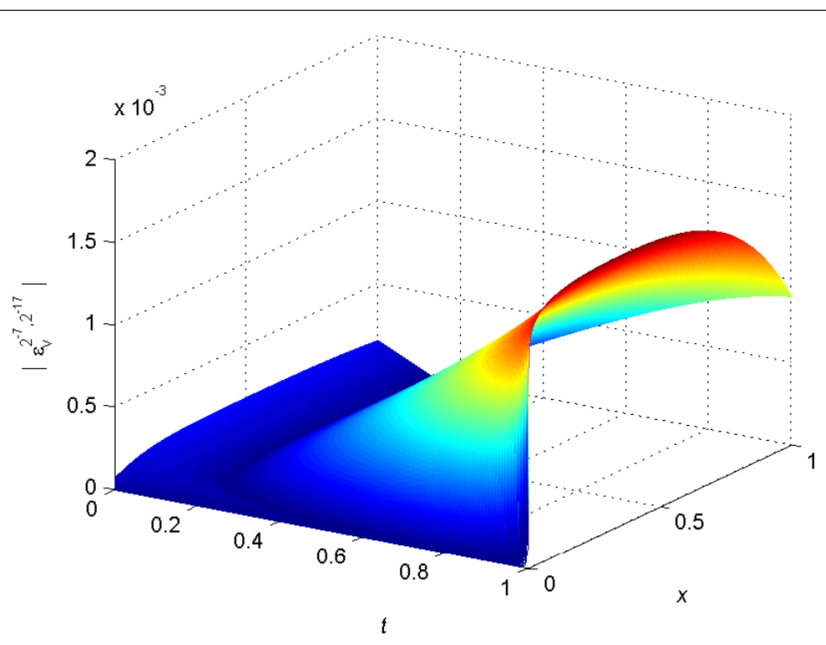

Figure 1 The error function $\left|\varepsilon_{v}^{2^{-7}, 2^{-17}}\right|=|\widetilde{v}-v|$ for $h=2^{-7}$, and $\tau=2^{-17}$ for Example 1

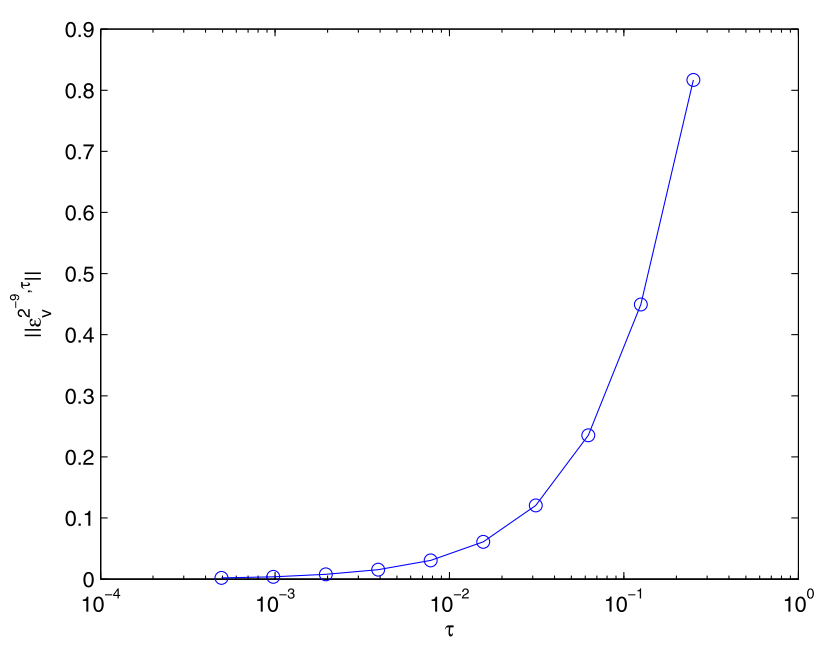

Figure 2 The maximum errors $\left\|\varepsilon_{V}^{2^{-9}, \tau}\right\|$ for $h=2^{-9}$, with respect to $\tau$ of Example 1

$$
\begin{aligned}
& u(0, t)=\frac{5}{18} t^{\frac{18}{5}} \quad \text { on } \gamma_{1} \\
& u(1, t)=\frac{5}{18} t^{\frac{18}{5}}+\frac{5}{36} \frac{5}{18} \cos \left(t^{\frac{18}{5}}\right)+1 \quad \text { on } \gamma_{3}
\end{aligned}
$$




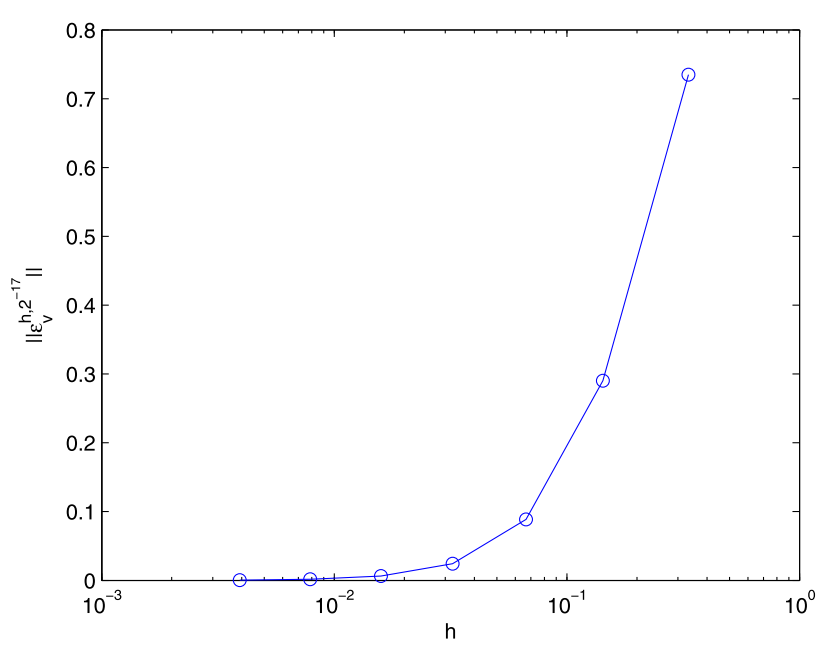

Figure 3 The maximum errors $\left\|\varepsilon_{v}^{h, 2^{-17}}\right\|$ for $\tau=2^{-17}$, with respect to $h$ of Example 1

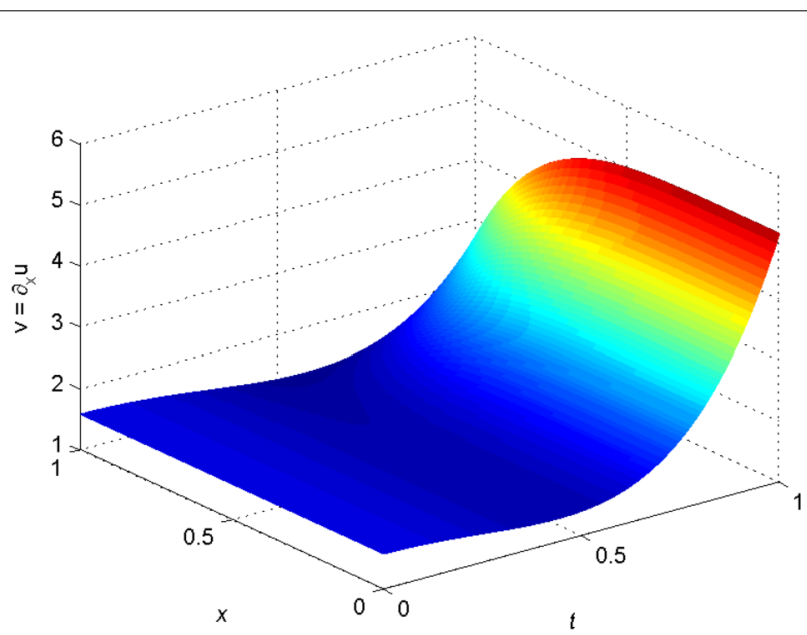

Figure 4 The function $v$ presenting the exact solution $\partial_{x} u$ for Example 1

where $f(x, t)=-\frac{5}{36} x^{\frac{36}{5}} t^{\frac{13}{5}} \sin \left(t^{\frac{18}{5}}\right)+t^{\frac{13}{5}}-\frac{31}{18} x^{\frac{26}{5}} \cos \left(t^{\frac{18}{5}}\right)+\frac{\pi^{2}}{4} \sin \left(\frac{\pi x}{2}\right)$. Using the proposed implicit six point difference scheme (28)-(30) $(\varrho=6)$ we obtain the approximate solution $\tilde{u}$ by applying the Gauss-Thomas method [12] for solving algebraic system of equations at each time level for $r=2^{-\omega}$ where $\omega$ is nonnegative integer. Next the boundary value problem for $v=\frac{\partial u}{\partial x}$ is constructed from the proposed Problem 2(ii) using the obtained approximate solution $\widetilde{u}$. Furthermore, the approximate solution $\widetilde{v}$ for $\frac{\partial u}{\partial x}$ is obtained at the same grid points by solving the system of equations using (94)-(96), and compared on the grids with the known exact solution $v(x, t)=\frac{5}{18} x^{\frac{31}{5}} \cos \left(t^{\frac{18}{5}}\right)+\frac{\pi}{2} \cos \left(\frac{\pi x}{2}\right)$. We use

$$
\widetilde{\Re}_{v}^{h, \tau}=\frac{\left\|\varepsilon_{v}^{2^{-\mu}, 2^{-\lambda}}\right\|}{\left\|\varepsilon_{v}^{2^{-(\mu+1)}, 2^{-(\lambda+1)}}\right\|}
$$

to present the order of convergence of $\widetilde{v}$ with respect to $h$ and $\tau$. Note that $O\left(h^{2}+\tau^{2}\right)$ order of convergence corresponds to $\asymp 2^{2}$ of the quantity by (116). Table 3 shows the 


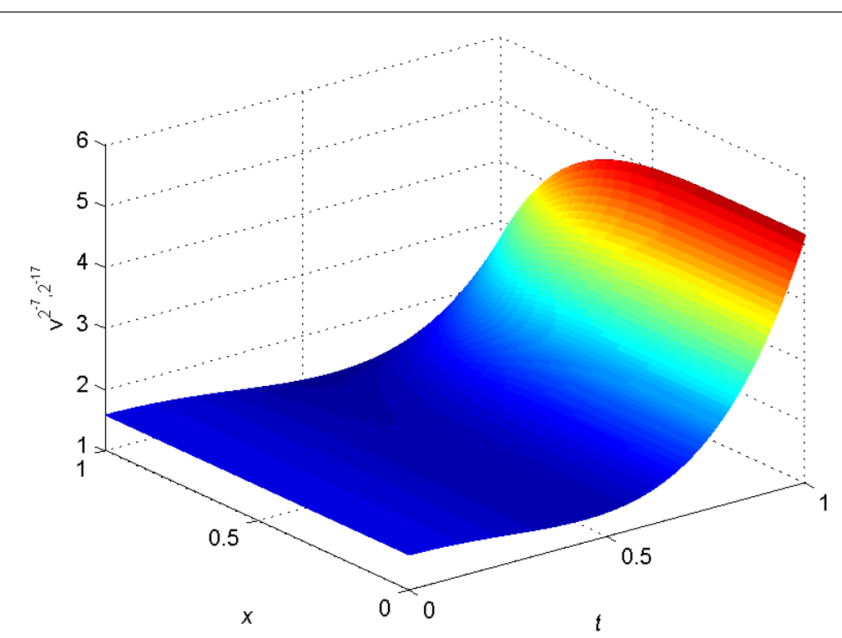

Figure 5 The grid function $v^{2^{-7}, 2^{-17}}$ presenting the approximate solution $\widetilde{v}$ of $\partial_{x} u$ when $h=2^{-7}, \tau=2^{-17}$ for Example 1

Table 3 Maximum errors and the order of convergence of approximate solution $\tilde{v}$ with respect to $h$ and $\tau$ for Example 2

\begin{tabular}{lllll}
\hline$\left(h=2^{-\mu}, \tau=2^{-\lambda}\right)$ & $\left(h=2^{-(\mu+1)}, \tau=2^{-(\lambda+1)}\right)$ & $\left\|\varepsilon_{V}^{2^{-\mu}, 2^{-\lambda}}\right\|$ & $\left\|\varepsilon_{V}^{2^{-(\mu+1)}, 2^{-(\lambda+1)}}\right\|$ & $\tilde{\Re}_{V}^{h, \tau}$ \\
\hline$\left(2^{-4}, 2^{-13}\right)$ & $\left(2^{-5}, 2^{-14}\right)$ & $1.0567 \mathrm{E}-2$ & $3.1449 \mathrm{E}-3$ & 3.360 \\
$\left(2^{-5}, 2^{-14}\right)$ & $\left(2^{-6}, 2^{-15}\right)$ & $3.1449 \mathrm{E}-3$ & $8.5353 \mathrm{E}-4$ & 3.685 \\
$\left(2^{-6}, 2^{-15}\right)$ & $\left(2^{-7}, 2^{-16}\right)$ & $8.5353 \mathrm{E}-4$ & $2.2209 \mathrm{E}-4$ & 3.843 \\
$\left(2^{-7}, 2^{-16}\right)$ & $\left(2^{-8}, 2^{-17}\right)$ & $2.2209 \mathrm{E}-4$ & $5.6628 \mathrm{E}-5$ & 3.922 \\
\hline
\end{tabular}

maximum errors for $h=2^{-\mu}, \mu=4,5,6,7,8$ and $\tau=2^{-\lambda}, \lambda=13,14,15,16,17$, respectively, and the orders $\widetilde{\Re}_{v}^{h, \tau}$ for Example 2. The third and fourth columns of this table present the theoretical upper bound errors given in (97). Figure 6 presents the error function $\left|\varepsilon_{v}^{2^{-7}, 2^{-17}}\right|$ for $h=2^{-7}$, and $\tau=2^{-17}$ for Example 2. The maximum errors $\left\|\varepsilon_{v}^{h, 2^{-17}}\right\|$ when $\tau=2^{-17}$, with respect to $h$, is demonstrated by Fig. 7 for Example 2. Figure 8 shows the exact solution $v(x, t)=\partial_{x} u$, and the grid function $v^{2^{-7}, 2^{-17}}$ presenting the approximate solution $\widetilde{v}$ of $\partial_{x} u$ when $h=2^{-7}, \tau=2^{-17}$ for Example 2.

\section{Example 3}

$$
\begin{aligned}
& L u=f(x, t) \quad \text { on } Q_{T}, \\
& u(x, 0)=e^{-x} \text { on } \gamma_{2}, \\
& u(0, t)=1+0.001 t^{\frac{25}{7}} \text { on } \gamma_{1}, \\
& u(1, t)=0.0001 \sin \left(t^{\frac{25}{7}}\right)+0.001 t^{\frac{25}{7}}+e^{-1} \text { on } \gamma_{3},
\end{aligned}
$$

where $f(x, t)=0.0001 \frac{25}{7} x^{\frac{50}{7}} t^{\frac{18}{7}} \cos \left(t^{\frac{25}{7}}\right)+0.001 \frac{25}{7} t^{\frac{18}{7}}-0.0001 \frac{50}{7} \frac{43}{7} x^{\frac{36}{7}} \sin \left(t^{\frac{25}{7}}\right)-e^{-x}$. The initial function, the boundary functions and the nonhomogeneous term $f(x, t)$ in Example 3 satisfy the conditions (21) of Problem 1(ii). Using the proposed implicit six point difference problem (28)-(30) $(\varrho=6)$ we obtain the approximate solution $\widetilde{u}$ at each time level. Next the boundary value problem for $v=\frac{\partial u}{\partial x}$ is constructed from the proposed Problem 2(ii) us- 


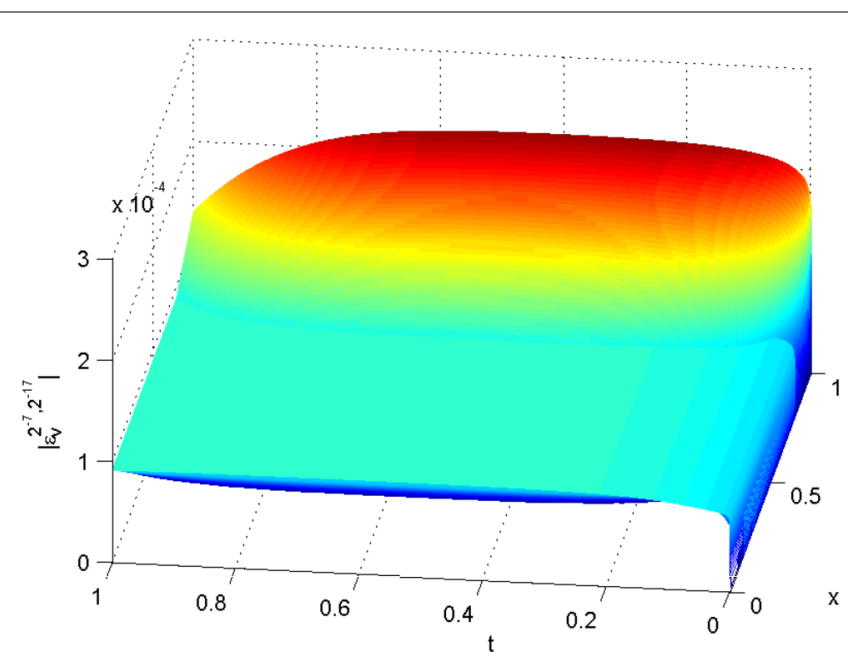

Figure 6 The error function $\left|\varepsilon_{v}^{2^{-7}, 2^{-17}}\right|=|\widetilde{v}-v|$ when $h=2^{-7}$, and $\tau=2^{-17}$ for Example 2

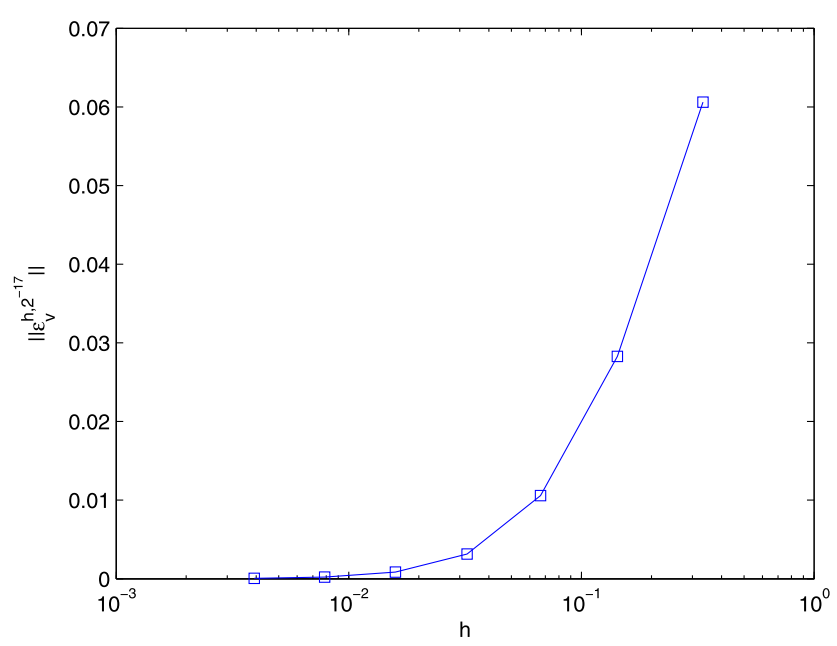

Figure 7 The maximum errors $\left\|\varepsilon_{v}^{h, 2^{-17}}\right\|$ for $\tau=2^{-17}$, with respect to $h$ for Example 2

ing the obtained approximate solution $\widetilde{u}$; then the approximate solution $\widetilde{v}$ of $v=\partial_{x} u$ is obtained at the same grid points by solving the system of equations resulting from (94)-(96). Let $v^{2^{-\mu}, 2^{-\lambda}}(x, t)$ be the approximate solution $\widetilde{v}$ at $(x, t)$ when $h=2^{-\mu}$ and $\tau=2^{-\lambda}$. The exact solution $v$ is not given. To verify the order of convergence of the computed solution $\widetilde{v}$ to the exact solution $v$ we compute the solution at grid points with successively reduced step sizes $h$ and $\tau$ by a factor of two and the ratio of the absolute successive errors (see Chap. 2 of [13]). Table 4 presents $v^{2^{-\mu}, 2^{-\lambda}}(x, t)$ at the grid points $(0.125,1),(0.25,1),(0.375,1),(0.5,1)$, $(0.625,1),(0.75,1)$ and $(0.875,1)$ for the pairs $(\mu, \lambda)=(5,13),(6,14),(7,15),(8,16$,$) which$ means that the step sizes $h$ in $x$ and $\tau$ in $t$ are halved successively. Table 5 demonstrates the absolute error ratios

$$
r_{1}=\left|\frac{v^{2^{-5}, 2^{-13}}(x, 1)-v^{2^{-6}, 2^{-14}}(x, 1)}{v^{2^{-6}, 2^{-14}}(x, 1)-v^{2^{-7}, 2^{-15}}(x, 1)}\right|,
$$




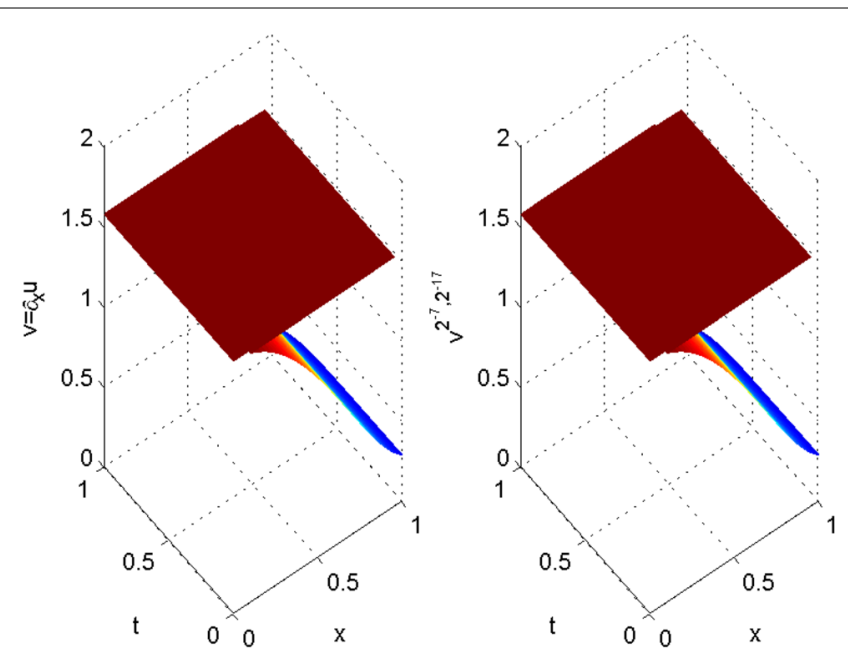

Figure 8 The exact solution $\partial_{x} u$ and the grid function $v^{2^{-7}, 2^{-17}}$ for $h=2^{-7}, \tau=2^{-17}$ of Example 2

Table 4 The approximate solution $\tilde{v}$ at some grid points on $t=1$ for Example 3

\begin{tabular}{lllll}
\hline$x$ & $v^{2^{-5}, 2^{-13}}(x, 1)$ & $v^{2^{-6}, 2^{-14}}(x, 1)$ & $v^{2^{-7}, 2^{-15}}(x, 1)$ & $v^{2^{-8}, 2^{-16}}(x, 1)$ \\
\hline 0.125 & -0.88218321 & -0.88241771 & -0.88247701 & -0.88249191 \\
0.25 & -0.77852029 & -0.77872997 & -0.77878291 & -0.77879622 \\
0.375 & -0.68703982 & -0.68722539 & -0.68727216 & -0.68728390 \\
0.5 & -0.60630576 & -0.60646779 & -0.60650853 & -0.60651874 \\
0.625 & -0.53504253 & -0.53518148 & -0.53521631 & -0.53522502 \\
0.75 & -0.47210904 & -0.47222525 & -0.47225424 & -0.47226147 \\
0.875 & -0.41647280 & -0.41656648 & -0.41658968 & -0.41659545 \\
\hline
\end{tabular}

$$
r_{2}=\left|\frac{v^{2^{-6}, 2^{-14}}(x, 1)-v^{2^{-7}, 2^{-15}}(x, 1)}{v^{2^{-7}, 2^{-15}}(x, 1)-v^{2^{-8}, 2^{-16}}(x, 1)}\right|,
$$

and the corresponding orders

$$
\begin{aligned}
& p_{1}=\log _{2}\left|\frac{v^{2^{-5}, 2^{-13}}(x, 1)-v^{2^{-6}, 2^{-14}}(x, 1)}{v^{2^{-6}, 2^{-14}}(x, 1)-v^{2^{-7}, 2^{-15}}(x, 1)}\right|, \\
& p_{2}=\log _{2}\left|\frac{v^{2^{-6}, 2^{-14}}(x, 1)-v^{2^{-7}, 2^{-15}}(x, 1)}{v^{2^{-7}, 2^{-15}}(x, 1)-v^{2^{-8}, 2^{-16}}(x, 1)}\right|,
\end{aligned}
$$

for the considered points at $t=1$. By analyzing the values of $p_{1}$ and $p_{2}$ in the third and fifth columns of Table 5 , respectively, we conclude that the order of convergence is quadratic in the two variables $x$ and $t$ on $t=1$. Figure 9 illustrates the grid function $v^{2^{-8}, 2^{-16}}$ presenting the approximate solution $\widetilde{v}$ of $v=\partial_{x} u$ when $h=2^{-8}, \tau=2^{-16}$ for Example 3.

\section{Concluding remarks}

Special difference problems of four point and six point implicit schemes for the first derivative of the solution $u(x, t)$ of the first type boundary value problem for a one dimensional heat equation with respect to the spatial variable $x$ are given. It is assumed that the initial function, boundary functions and the nonhomogeneous term in the heat equation possess 
Table 5 The absolute error ratios at some grid points on $t=1$ and the orders $p_{1}, p_{2}$ for Example 3

\begin{tabular}{lllll}
\hline$x$ & $r_{1}$ & $p_{1}$ & $r_{2}$ & $p_{2}$ \\
\hline 0.125 & 3.9544688 & 1.9835 & 3.9798658 & 1.9927 \\
0.25 & 3.9607102 & 1.9858 & 3.9774606 & 1.9919 \\
0.375 & 3.9675005 & 1.9882 & 3.9838160 & 1.9942 \\
0.5 & 3.9777172 & 1.9917 & 3.9902057 & 1.9965 \\
0.625 & 3.9893770 & 1.9962 & 3.9988519 & 1.9996 \\
0.75 & 4.0086237 & 2.0031 & 4.0096819 & 2.0035 \\
0.875 & 4.0379310 & 2.0136 & 4.0207972 & 2.0075 \\
\hline
\end{tabular}

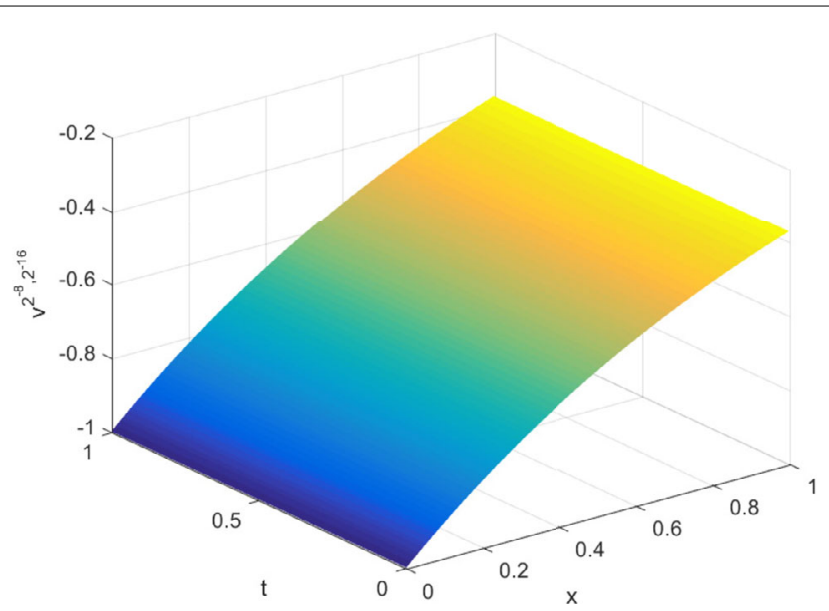

Figure 9 The grid function $v^{2^{-8}, 2^{-16}}$ presenting the approximate solution $\widetilde{v}$ of $\partial_{x} u$ when $h=2^{-8}, \tau=2^{-16}$ for Example 3

a number of derivatives in the variables $x$ and $t$ necessary in this connection for performing current and subsequent manipulation in approximating $\frac{\partial u}{\partial x}$. We prove that the solution of the proposed four point and six point difference schemes converge to the exact value of $\frac{\partial u}{\partial x}$ on the grids of order $O\left(h^{2}+\tau\right)$ and $O\left(h^{2}+\tau^{2}\right)$, respectively.

Remark 12 These results can be used in some domain decomposition methods allowing for parallel computation [14, 15]. Furthermore, the proposed approach may be applicable to similar equations, given in the phenomena of impact of a moving foot on the transfer of heat from a constantly heated warm water into the foot immersed within a footbath [16] and the enhancement of performance by increasing the thermal efficiency of a direct absorption solar collector based on an alimino-water nanofluid [17].

Remark 13 The proposed approach can also be applied to finding second order derivatives of the solution of the first type boundary value problem for a one dimensional heat equation and this research will be presented in a subsequent article. Also the methodology may be extended to a two dimensional heat equation.

Acknowledgements

The authors thank Professor A.A. Dosiyev for his attention to this work and valuable advices. 
Competing interests

The authors declare that they have no competing interests.

Authors' contributions

The authors contributed equally to the writing of this paper. All authors read and approved the final manuscript.

\section{Publisher's Note}

Springer Nature remains neutral with regard to jurisdictional claims in published maps and institutional affiliations.

Received: 13 February 2018 Accepted: 12 November 2018 Published online: 27 November 2018

\section{References}

1. Bateman, H.: Partial Differential Equations of Mathematical Physics. Dover, New York (1944)

2. Tuttle, F.: A mathematical theory of the drying of wood. J. Franklin Inst. 200, 609-614 (1925)

3. Volkov, E.A.: On convergence in $C_{2}$ of a difference solution of the Laplace equation on a rectangle. Russ. J. Numer. Anal. Math. Model. 14(3), 291-298 (1999)

4. Dosiyev, A.A., Sadeghi, M.M.H.: A fourth order accurate approximation of the first and pure second derivatives of the Laplace equation on a rectangle. Adv. Differ. Equ. 2015, Article ID 67 (2015). https://doi.org/10.1186/s13662-015-0408-8

5. Volkov, E.A.: On the grid method by approximating the derivatives of the solution of the Dirichlet problem for the Laplace equation on the rectangular parallelpiped. Russ. J. Numer. Anal. Math. Model. 19(3), 209-278 (2004)

6. Dosiyev, A.A., Sadeghi, M.M.H.: On a highly accurate approximation of the first and pure second derivatives of the Laplace equation in a rectangular parellelpiped. Adv. Differ. Equ. 2016, Article ID 145 (2016). https://doi.org/10.1186/s13662-016-0868-5

7. Dosiyev, A.A., Abdussalam, A.: On the high order convergence of the difference solution of Laplace's equation in a rectangular parallelepiped. Filomat 32(3), 893-901 (2018)

8. Dosiyev, A.A., Sarikaya, H.: 14-point difference operator for the approximation of the first derivatives of a solution of Laplace's equation in a rectangular parallelepiped. Filomat 32(3), 791-800 (2018)

9. Samarskii, A.A.: The Theory of Difference Schemes. Dekker, New York (2001)

10. Ladyženskaja, O.A., Solonnikov, V.A., Ural'ceva, N.N.: Linear and Quasi-Linear Equations of Parabolic Type. Translation of Mathematical Monographs, vol. 23. Am. Math. Soc, Providence (1967)

11. Burden, R.L., Faires, J.D.: Numerical Analysis. Cengage Learning, Boston (2011)

12. Fausett, L.V.: Applied Numerical Analysis Using Matlab. Pearson Prentice Hall, Upper Saddle River (2008)

13. Einarsson, B.: Accuracy and Reliability in Scientific Computing. SIAM, Philadelphia (2005)

14. Kuznetsov, Y.: New algorithms for approximate realization of implicit difference schemes. Sov. J. Numer. Anal. Math. Model. 3(2), 99-114 (1998). https://doi.org/10.1515/rnam.1988.3.2.99

15. Dawson, C.N., Du, Q., Dupont, T.F.: A finite difference domain decomposition algorithm for numerical solution of the heat equation. Math. Comput. 57(195), 63-71 (1991)

16. Turkyilmazoglu, M.: Heat transfer from warm water to a moving foot in a footbath. Appl. Therm. Eng. 98, 280-287 (2016)

17. Turkyilmazoglu, M.: Performance of direct absorption solar collector with nanofluid mixture. Energy Conserv. Manag. $114,1-10(2016)$

\section{Submit your manuscript to a SpringerOpen ${ }^{\odot}$ journal and benefit from:}

- Convenient online submission

- Rigorous peer review

- Open access: articles freely available online

- High visibility within the field

- Retaining the copyright to your article

Submit your next manuscript at $\boldsymbol{~ s p r i n g e r o p e n . c o m ~}$ 